\title{
Preventing the exacerbation of health disparities by iatrogenic pharmacogenomic applications: lessons from warfarin
}

\author{
Jorge Duconge*,1 \& Gualberto Ruaño² \\ ${ }^{1}$ Pharmaceutical Sciences Department, School of Pharmacy, University of Puerto Rico Medical Sciences Campus, PO Box 365067 , \\ San Juan, PR 00936, USA \\ ${ }^{2}$ Institute of Living at Hartford Hospital, Hartford, CT 06102, USA \\ *Author for correspondence: jorge.duconge@upr.edu \\ 'GHealth disparities could be exacerbated when polymorphisms and algorithms are not suitable to \\ populations with ethnogeographic particularities"
}

First draft submitted: 11 April 2018; Accepted for publication: 30 May 2018; Published online: 14 June 2018

Keywords: African-Americans $\bullet$ ethnicity $\bullet$ health disparity $\bullet$ Hispanics $\bullet$ race $\bullet$ warfarin pharmacogenomics

Pharmacogenomics interventions in healthcare are used to guide and select pharmacotherapies based on individual polymorphism and interpretation algorithms. Like any intervention, there is a balance of benefit versus risk that needs to be assessed. The more complete the polymorphism detection and the more learned the algorithms, the better benefit [1]. Conversely, limited polymorphism and untested algorithms increase the risk. Health disparities could be exacerbated when polymorphisms and algorithms are not suitable to populations with ethnogeographic particularities. We detail examples for African-American and Hispanic populations that point to potential harm from pharmacogenomics. We then posit various measures to prevent it. We have raised the issues of pharmacogenomic research in ethnic minorities to prevent health disparities and enhance health equity.

Warfarin has been the 'poster child' of pharmacogenetics for the realities of healthcare. This historical drug has been rejuvenated by a number of pharmacogenetics firsts. It is the first and only drug with the US FDA label encompassing pharmacokinetic (CYP2C9) and pharmacodynamic (VKORC1) genes. Warfarin was the first drug to have multiple FDA-approved in vitro diagnostic kits for clinical implementation, and it was the first pharmacogenomic system to confront scrutiny by payers including Medicare. The decision was to approve reimbursement only under 'evidence development' in clinical trials. Warfarin was also the first pharmacogenomic system to have economic analysis of potential savings return on investment and number needed to treat. In this regard, warfarin continues at the avant garde of the field with new research on African-American and Hispanic patients.

Related to the thesis of this article, warfarin was also the first pharmacogenomic system (genotyping, algorithm, clinical decision support) to be examined in a prospective trial (Clarification of Optimal Anticoagulation through Genetics [COAG]). One of the observations was the African-American cohort performed worse than the control. Currently, there is a large array of population-derived pharmacogenetics-guided dosing algorithms that on average explained approximately between 35 and $75 \%$ of the interpatient variability in warfarin dose requirements [214]. Usually, these algorithms are derived from cohorts of mostly European patients and, therefore, only the common CYP2C9*2, CYP2C9*3 and VKORC1-1639G $>\mathrm{A}$ variants - with CYP4F2*3 to a lesser extent - are included. Notably, the power of each validated and published genotype-driven algorithm to capture most of the observed dose variance at a given population will depend on the availability of relevant data from all ethnic groups encompassing the genomic diversity within the population. Unfortunately, the performance of these pharmacogenetic-guided algorithms has only been marginally studied in Hispanics, a minority population that is often excluded or underrepresented in clinical studies. Accordingly, there is still a gap of knowledge regarding the impact of pharmacogenomics on dose requirements for admixed Hispanics.

Future Medicine 


\section{Hispanic studies \\ Puerto Rico}

In 2009, our group analyzed genomic DNA specimens from the local Newborn Screening Program in order to ascertain the frequency distribution of the major pharmacogenetic predictors of warfarin dose requirements in the Puerto Rican population. The estimated frequencies for common alleles CYP2C9*2, CYP2C9*3 and VKORC11639 G $>A$ in newborns from Puerto Rico were 6.5, 5.3 and 28.8\%, respectively [15]. These frequencies were lower than those in Puerto Ricans on warfarin from a clinical pharmacogenetic study conducted at an anticoagulation clinic in San Juan, Puerto Rico (10.0, 6.0 and 36.0\%, respectively) [12]. On the other hand, Puerto Ricans from the 1000 Genomes project have slightly different frequencies: $14.0,4.0$ and 39.0\% for the above mentioned alleles [16].

We also assessed the association between major genetic polymorphisms occurring on relevant pharmacogenes and the incidence of adverse events in Puerto Rican patients (i.e., major bleeding events, longer time to achieve stabilization of anticoagulation therapy and overanticoagulation, international normalized ratio [INRs] >4) [17]. Although no significant association was found between genotypes and individual outcomes over the first 90 days of therapy, carriers of CYP2C9 and VKORC1 variants combined showed a higher incidence of multiple adverse events (HR: 2.5; 95\% CI: $1.0-6.3 ; \mathrm{p}=0.04$ ). In another study, we compared the performance of a Puerto Rican-oriented genotype-guided algorithm to predict warfarin doses in this population versus different published pharmacogenetics algorithms (e.g., International Warfarin Pharmacogenetics Consortium [IWPC], Lenzini et al. [9]), a clinically-guided algorithm and the fixed-dose method [12]. We found that the clinical algorithm explained essentially the same percentage of variability in Puerto Rican patients with low dose requirements $(\leq 3 \mathrm{mg} /$ day $)$ when compared with the pharmacogenetic algorithms $\left(R^{2}=49\right.$ vs $\left.48.4 \%\right)$, but the prediction errors were smaller with the genotype-guided models [12]. Overall, we demonstrated that previously published pharmacogenetics-driven dosing algorithms showed better dose predictions than a clinical approach in Puerto Rican patients with warfarin dose requirements lower than $3 \mathrm{mg} /$ day (sensitives) but not in patients with either intermediate- or high-dose requirements. A limitation of these pharmacogenetics algorithms, including the one originally developed in Puerto Ricans, was that they all relied on common alleles CYP2C9*2, CYP2C9*3 and VKORC1 $-1639 \mathrm{G}>\mathrm{A}$.

In order to address this issue, we recently published our findings from case-control pharmacogenetic association studies in 275 Puerto Rican patients on warfarin $[10,11]$. An admixture-adjusted pharmacogenetics-guided algorithm that explained two-thirds of observed variance in stable warfarin dose in this Caribbean Hispanic population was developed. Besides, the CYP4F2 *3 and NQO1 *2 variants were independently associated with a 17 and $10 \%$ increase of the dose per allele, respectively. On the other hand, the admixture index decreases the dose by $7 \%$. The African-related rare $\mathrm{CYP}_{2} \mathrm{C9}^{*} 8$ allele explained $31 \%$ decrease of the dose. The genomic diversity of Puerto Ricans was highlighted by the presence of 11 major CYP2C9 haplotypes. The CYP2C9 rs2860905 variant was found for the first-time ever to be significantly associated with warfarin dose requirements and showed stronger association with warfarin sensitivity than common CYP2C9*2 and *3 alleles. Incorporation of rs 2860905 in a model that also includes additional genetics (i.e., VKORC1-1639G >A; CYP2C9 rs1856908; ABCB1 c.IVS9-44A >G; CES2 c.269-965A $>\mathrm{G}$ ) and nongenetic factors showed better prediction of warfarin dose requirements in this Caribbean Hispanic patients than previous genetic-driven algorithms [10,11]. We concluded that admixture and ethno-specific alleles are both clinically relevant predictors for algorithmically computed warfarin doses in Caribbean Hispanics.

\section{Brazilians \& other Latino populations}

An earlier study by Botton et al. confirmed that common polymorphisms on CYP2C9 and VKORC1 are the main predictors of warfarin dose in a southern Brazilian population of European ancestry, but they also postulated that other genes or genetic variants (e.g., F2 494C>T) with smaller effects can indeed improve the algorithm for better dose prediction [18]. A GWAS conducted in Brazilians and Europeans have identified two other variants in CYP2C9 associated with warfarin dose requirements that are in linkage disequilibrium (LD) with rs2860905 as well as CYP2C9 * 2 or *3. The top genome-wide signals in the CYP2C9 region were rs9332238 (odds ratio: 6.8; $\mathrm{p}=4.4$ $\times 10^{-13}$ ) and rs4917639 in the warfarin-treated Brazilians and Europeans cohorts, respectively [19,20]. Moreover, the lead SNP in the VKORC1 locus of Brazilians was rs749671 (odds ratio: 20.4; $\mathrm{p}=1.08 \times 10^{-33}$ ).

Noteworthy, CYP2C9 was one of the first clinically relevant pharmacogenes investigated by REFARGEN (Rede Nacional de Farmacogenetica/genomica de Brazil). A recent report by Rodrigues-Soares et al. from the RIBEF/EPIGEN-Brazil initiative found that CYP2C9*2 and VKORC1-1639G>A (rs9923231) alleles are more frequent in Whites than in Blacks Brazilians, whereas Brazilian native Americans showed lower frequencies of CYP2C9*2 [21]. Strikingly, they suggested that color categories as a social construct were four-times more informative 
of the variance of warfarin pharmaco-allele frequencies than geographic regions. However, both the REFARGEN and RIBEF initiatives favored the use of genetic markers to infer the biogeographic ancestry in pharmacogenetic studies. Interestingly, in the multiple regression model developed by Perini et al. that gave rise to the pharmacogeneticguided algorithm for predicting stable weekly warfarin dosing in Brazilians $\left(R^{2}=51 \% ; n=390\right)$, self-identified race or color categories was not found to be significantly associated with dose [22]. However, the required warfarin dose was significantly lower in White than in Black Brazilians (i.e., $29 \pm 13$ vs $35 \pm 15 \mathrm{mg} /$ week; $\mathrm{p}=0.006$ ). We strongly believe that genomic-based inferences of ancestry are more informative and accurate than the use of color/race labels as a proxy, which can even be distinctively interpreted across different regions. It is estimated that around 56\% of Brazilians (>90 million inhabitants) have more than 50\% African ancestry [23].

The prevalence of major CYP2C9 2 and $* 3$ alleles in Brazilians, stratified by color categories, are as follows: White Brazilians (12 and 8.1\%, respectively); Mestizos/Pardos (7.2 and 6.8\%, respectively) and Black Brazilians (4.5 and 3.2\%, respectively) [23,24]. In Brazilians, the frequency distribution of rs9923231 is best described as a continuous function of the individual proportions of African ancestry, irrespective of self-reported race or skin color [25]. A previous paper by Perini et al. showed that the VKORC1-1639G>A variant, also called 3673G $>$ A, has frequencies ranging from 40 to $60 \%$ in three Native American populations of Brazil [26]. Since Native Americans in Brazil comprise small groups that are often scattered across the entire country, allele frequencies published in a specific study should be observed with caution as they may not be representative of all Brazilian Native American and their allele frequencies may be significantly affected by genetic drift.

In Brown and Black Brazilians, the combined effect size of CYP2C9 $* 2$ and *3 amounted to average reductions of 5.1 and $9.4 \mathrm{mg} /$ weekly per variant allele, respectively. In addition to the well-known effect of the rs 9923231 promoter variant, lower warfarin requirements have also been associated with VKORC1 rs8050894 and rs2884737 in an admixed Brazilian cohort [22,25]. Accordingly, Suarez-Kurtz et al. argued that INR measurements are useful but cannot completely capture the information on dose variance provided by CYP2C9 and, particularly VKORC1 genotypes, the latter remaining the most informative predictor of stable warfarin dose requirements among Brazilians [27]. Interestingly, the VKORC1-1639G $>\mathrm{A}$ explained only $11 \%$ of variability in warfarin dose requirements among Colombians versus $20-30 \%$ that is normally explained among Europeans [28].

\section{African-American studies}

The COAG (ClinicalTrial.gov ID number NCT00839657) was a double-blinded and randomized clinical trial [14] conducted in a multi-ethnic cohort from the USA to compare a pharmacogenetic algorithm with a clinical method. The primary outcome was the percentage of time that INRs were within the therapeutic range for approximately 24 days. Although the authors reportedly found no improvements of the anticoagulation therapy after using individual genetic information, the genetic-based algorithm had significantly less predictability among patients self-identified as Blacks (presumably African-Americans) versus White Americans (presumably of European descent). Indeed, patients from the self-reported black race were more likely to have INRs over the therapeutic range, and it took longer on average for them to reach the first therapeutic INR when compared with those in the clinical algorithm. It should also be noted that 39 out of 501 patients experienced major bleeding events, thrombosis or died when treated with the clinically guided algorithm as compared with only 19 out of 514 in the genotype-guided dosing group [14]. The number of individuals that experienced major bleeding events through the end of follow-up period were significantly higher in the group receiving clinical dosing than in the one following the genetic-guided dosing recommendations (HR: 0.36; 95\%CI: 0.15-0.86; $\mathrm{p}=0.021$ ), which is further supporting the potential benefit of therapy guided by genetic information.

One important concern with the COAG trial design is that a genetic-guided dosing algorithm is compared against a so-called 'clinical algorithm' that is by no means the current standard of care for warfarin therapy [29]. In addition, the poor performance of the published genetic-guided dosing algorithms among self-identified Blacks in this trial was expected since the variants evaluated in the COAG trial (i.e., CYP2C9*2, CYP2C9*3 and VKORC1$1639 \mathrm{G}>\mathrm{A}$ ) are less common in patients of African origin than in Europeans, whereas important variants in Africans and their descendants were omitted (e.g., prevalence of CYP2C9*5, *6, *8, * 11 variants in Africans is $\sim 15-20 \%$; rs $12777823>40 \%)[1,30-33]$. This study limitation led to an overestimation of dose requirements among participants self-identified as Blacks. Strikingly, Drozda et al. found that the dosing prediction error with COAG trial algorithm in African-Americans was on average $2.2 \mathrm{mg} /$ day for carriers of the CYP2C9*5, CYP2C9*6, CYP2C9*8, CYP2C9*11 ( $\mathrm{p}<0.0001)$ and $2.0 \mathrm{mg} /$ day for rs12777823AA individuals $(\mathrm{p}<0.0001)$ [1]. Likewise, in spites of the fact that no differences were found between clinical and genotype COAG groups with regard to 
the percentage of time in range at 30 days, this parameter was significantly higher in self-identified Blacks patients who used the clinically guided dose recommendations in comparison to the genotype-guided algorithm $(\mathrm{p}=0.01)$, whereas the percentage of time with INR $>3$ was lower $(\mathrm{p}=0.004)$ in the same group. These findings support the idea of developing genetic-guided dosing algorithms that account for ethno-specific polymorphisms relevant to each ethnic group or population residing in a particular ethno-geographic region.

In contrast to the COAG trial, the European Pharmacogenetics of Anticoagulant Therapy trial (EU-PACT, NCT01119300) performed in European patients found slightly but significant improvement in the anticoagulation therapy when genetic information was used [34]. Noteworthy, both trials used pharmacogenetic algorithms that were developed with data from patients of mostly European ancestry and, therefore, included alleles commonly found in Caucasians but not in other ethnic groups. The EU-PACT Group reported that the percentage of time within the therapeutic INR range was higher in patients in the genotype-dosing arm. Furthermore, the genetic-guided dosing algorithm showed sustained benefit [34,35].

The Genetics Informatics Trial (GIFT, NCT01006733) of warfarin to prevent deep vein thrombosis (DVT) is the first clinical trial that investigated the benefits of pharmacogenetic-guided dosing based on clinical outcomes. Preliminary results presented at the 66th Annual Scientific Session of the American College of Cardiology (March 2017, DC, USA) suggested a superiority of the pharmacogenetics-guided algorithm over the standard of care, with $27 \%$ reduction of the primary endpoints when compared with the standard dosing [36]. This finding prompted a revision of the current Clinical Pharmacogenetics Implementation Consortium (CPIC) guidelines for pharmacogenetics-guided warfarin dosing [3] and a careful assessment of the reported clinical trial designs and results [37].

\section{Role of race \& ethnicity in model predictability}

Interestingly, the inclusion of self-reported race or ethnicity in certain pharmacogenetics algorithms does not seem to have a significant effect on model predictability of warfarin dose requirements [38-40]. One possible explanation is that the effect of race is already captured by the genetic variants included in the algorithms [13]. A study aimed at assessing predictability of genotype-guided dosing algorithms revealed that a race-stratified analysis has higher predictability than race-combined analysis and it is because the effect of genetic predictors differs by race [32]. CYP2C9*2 and CYP4F2 *3 were specifically relevant to European Americans while rs 12777823 was only relevant to African-Americans. In addition, VKORC1-1639G $>$ A was associated with a decrease in warfarin dose among both populations, but with a higher impact in Europeans.

The CPIC guidelines publish recommendations to help clinicians understand how better use the available genetic information and test results in order to optimize individual drug therapy [3]. The CPIC guidelines for warfarin have recommendations (rated as level A or strong) to individually calculate optimal doses by using validated and published pharmacogenetic-guided algorithms that relies on common genetic variants, when test results are available (i.e., VKORC1-1639G $>\mathrm{A}, C Y P 2 C 9^{*} 2$ and $C Y P 2 C 9^{*} 3$ ). It is important to realize that all these common variants have been clearly found to be significantly associated with warfarin dose requirements and the resulting pharmacogenetic-guided algorithms have been validated in many populations worldwide, but mostly of European origin. An update of the guideline added self-reported ancestry and certain ethno-specific alleles into the decisionmaking flowchart. That is, for patients with self-reported African ancestry, the guideline recommends further reductions by as much as $30 \%$ of the algorithmically calculated dose based on relevant ethno-specific alleles for this population (i.e., CYP2C9*5, CYP2C9*6, CYP2C9*8, CYP2C9*11 and rs12777823). On the other hand, increase dose by $5-10 \%$ is recommended for patients without self-reported African ancestry who are also carriers of the CYP4F2*3.

Based on these guideline recommendations, one might now expect that dose predictions following a pharmacogenetics-guided algorithm could be somehow harmful for a given population if the relevant information and genotypes for their ethno-specific alleles were unavailable. In fact, the guidelines recommend not using genotype-guided predictions in Blacks when CYP2C9 data lacks African-related alleles *5, *6, *8, *11. Furthermore, since ancestry information is self-reported and not genetically inferred, it is unclear how this approach will improve predictability in admixed populations like Caribbean Hispanics, where self-reported race or skin color used as a proxy of their ancestry is often misleading [29]. We urge caution on the generalization of pharmacogenomics predictive systems to global health. Clinical studies must be representative of the target populations and enhanced diversity of participants should be the standard $[10,11,29,41]$. Quantification of ancestry and ethnicity by genetic 
markers should supersede definition based on physical appearance. Only then will personalized medicine become an integral part of global health and a tool toward health equity.

A warfarin-dosing strategy for a diverse population that accounts for the complexity of race and leverages electronic health records for relevant variables has been published in order to facilitate multiethnic algorithmguided predictions using preemptive genetic testing. Notably, this approach incorporates patient's genetics only when evidence to support its clinical actionability is available in the specific patient population [42].

\section{Additional pitfalls}

In approaching predictions of drug responsiveness, algorithms should include gene-gene and gene-environment interactions, as well as other descriptors of the patient's baseline. Personalizing drug treatment requires screening for warfarin genetic alterations in the patient but also interfacing environmental triggers which interacts with the gene targets. Drug and diet interactions with metabolism inhibitors and inducers are the preeminent environmental modifier in the pharmacokinetics dimension. Co-prescription of any strong CYP2C9 inhibitors could generate an equivalent dysfunctional phenotype and should be safeguarded with caution when warfarin is being prescribed. Conversely, induction of CYP2C9 by rifampin could lead to warfarin dose being increased to compensate for the induction, and then the drug may enhance risk of bleeding when the CYP2C9 inducer is discontinued. These are general safeguards on drug interactions which reduce the predictability of all algorithms. Strict reliance on algorithms without considering these interactions may be adverse in situations where the healthcare system is not routinely conducting surveillance for these modifiers.

There is promising research indicating that mathematical models other than linear regression may yield more predictive algorithms. Notably, the arena of big data, machine learning, artificial intelligence and neural networks offers new dimension in modeling of biological phenomena and gene-drug interactions. However, including more genes in a model does not necessarily mean more precise and better predictions. As the number of genes is increased, so do the roster of possible disease comorbidities and environmental modifiers which may confound gene effects. Hence, multigene models invariably require very large populations to validate.

\section{Acknowledgements}

The authors want to thank UPR-MSC RCMI Center for Genomics in Health Disparities and Rare Disorders for their support.

\section{Financial \& competing interest disclosure}

G Ruaño is founder and President of Genomas, Inc. The material presented herein is the result of work supported in part by the NIH grant number SC1 HL123911, from the National Heart, Lung and Blood Institute (NHLBI), as part of the SCORE MBRS Program supported by the National Institute of General Medical Sciences (NIGMS), and number U54 MD007600 from the National Institute on Minority Health and Health Disparities (NIMHD), as part of the Research centers in Minority Institutions (RCMI) Program. The authors have no other relevant affiliations or financial involvement with any organization or entity with a financial interest in or financial conflict with the subject matter or materials discussed in the manuscript apart from those disclosed.

No writing assistance was utilized in the production of this manuscript.

\section{Disclaimer}

The contents of this publication are solely the responsibility of the authors and do not represent the official views of the National Institutes of Health $(\mathrm{NIH})$ or the United States Government.

\section{References}

1 Drozda K, Wong S, Patel SR et al. Poor warfarin dose prediction with pharmacogenetic algorithms that exclude genotypes important for African Americans. Pharmacogenet. Genomics 25(2), 73-81 (2015).

2 Wu AHB, Wang P, Smith A et al. Dosing algorithm for warfarin using CYP2C9 and VKORC1 genotyping from a multi-ethnic population: comparison with other equations. Pharmacogenomics 9, 169-178 (2008).

3 Johnson JA, Caudle KE, Gong L et al. Clinical Pharmacogenetics Implementation Consortium (CPIC) guideline for pharmacogenetics-guided warfarin dosing: 2017 update. Clin. Pharmacol. Ther. 102(3), 397-404 (2017).

4 Reynolds K, Valdes R Jr, Hartung B, Linder M. Individualizing warfarin therapy. Per. Med. 4, 11-31 (2007).

5 Cavallari LH, Momary KM, Patel SR, Shapiro NL, Nutescu E, Viana MG. Pharmacogenomics of warfarin dose requirements in Hispanics. Blood Cells Mol. Dis. 46(2), 147-150 (2011).

6 Caldwell MD, Berg RL, Kai QZ et al. Evaluation of genetic factors for warfarin dose prediction. Clin. Med. Res. 5(1), 8-16 (2007). 
7 Gage BF, Eby C, Johnson JA et al. Use of pharmacogenetic and clinical factors to predict the therapeutic dose of warfarin. Clin. Pharmacol. Ther. 84(10), 326-331 (2009).

8 The International Warfarin Pharmacogenetics Consortium. Estimation of the warfarin dose with clinical and pharmacogenetic data. $N$ Engl. J. Med. 360(8), 753-764 (2009).

9 Lenzini P, Wadelius M, Kimmel S et al. Integration of genetic, clinical, and INR data to refine warfarin dosing. Clin. Pharmacol. Ther. 87(5), 572-578 (2010).

10 Claudio-Campos K, Labastida A, Ramos A et al. Warfarin anticoagulation therapy in Caribbean Hispanics of Puerto Rico: a candidate gene association study. Front. Pharmacol. 8, 347 (2017).

11 Duconge J, Ramos AS, Claudio-Campos $\mathrm{K}$ et al. A novel admixture-based pharmacogenetic approach to refine warfarin dosing in Caribbean Hispanics. PLoS ONE 11(1), e0145480 (2016).

12 Ramos AS, Seip RL, Rivera-Miranda G et al. Development of a pharmacogenetic-guided warfarin dosing algorithm for Puerto Rican patients. Pharmacogenomics 13(16), 1937-1950 (2012).

13 Lubitz SA, Scott SA, Rothlauf EB et al. Comparative performance of gene-based warfarin dosing algorithms in a multiethnic population. J. Thromb. Haemost. 8(5), 1018-1026 (2010).

14 Kimmel SE, French B, Kasner SE et al. A pharmacogenetic versus a clinical algorithm for warfarin dosing. N. Engl. J. Med. 369(24), 2283-2293 (2013).

15 Duconge J, Cadilla C, Windemuth A et al. Prevalence of combinatorial CYP2C9 and VKORC1 genotypes in Puerto Ricans: implications for warfarin management in Hispanics. Ethn. Dis. 19(4), 390-395 (2009).

16 The 1000 Genomes Project Consortium. A global reference for human genetic variation. Nature 526, 68-74 (2015).

17 Valentín II, Rivera G, Nieves-Plaza M et al. Pharmacogenetic association study of warfarin safety endpoints in Caribbean Hispanics. PR Health Sci. J. 33(3), 97-104 (2014).

18 Botton MR, Bandinelli E, Rohde LE, Amon LC, Hutz MH. Influence of genetic, biological and pharmacological factors on warfarin dose in a southern Brazilian population of European ancestry. Br. J. Clin. Pharmacol. 72, 442-450 (2011).

19 Takeuchi F, McGinnis R, Bourgeois $S$ et al. A genome-wide association study confirms VKORC1, CYP2C9, and CYP4F2 as principal genetic determinants of warfarin dose. PLoS Genet. 5, e1000433 (2009).

20 Parra EJ, Botton MR, Perini JA et al. Genome-wide association study of warfarin maintenance dose in a Brazilian sample. Pharmacogenomics 16(11), 1253-1263 (2015).

21 Rodrigues-Soares F, Kehdy FSG, Sampaio-Coelho J et al. Genetic structure of pharmacogenetic biomarkers in Brazil inferred from a systematic review and population-based cohorts: a RIBEF/EPIGEN-Brazil initiative. Pharmacogenomics J. 5(3), doi:10.1038/s41397-018-0015-7 (2018) (Epub ahead of print).

22 Perini JA, Struchiner CJ, Silva-Assunção E et al. Pharmacogenetics of warfarin: development of a dosing algorithm for Brazilian patients. Clin. Pharmacol. Ther. 84(6), 722-728 (2008).

23 Pena SD, Bortolini MC. Pode a genetica definir quem deve se beneficiar das cotas universitarias e demais acoes afirmativas? Estudos Avancados 18, 31-50 (2004).

24 Suarez-Kurtz G. Pharmacogenomics of admixed populations. Trends Pharmacol. Sci. 26(4), 196-201 (2005).

25 Suarez-Kurtz G, Bottom MR. Pharmacogenomics of warfarin in populations of African descent. Br. J. Clin. Pharmacol. 75(2), 334-346 (2012)

26 Perini JA, Petzl-Erler ML, Tsuneto LT, Suarez-Kurtz G. VKORC1 polymorphisms in Amerindian populations of Brazil. Pharmacogenomics 9(11), 1623-1629 (2008).

27 Suarez-Kurtz G, Perini JA, Silva-Assunção E, Struchiner CJ. Relative contribution of VKORC1, CYP2C9, and INR response to warfarin stable dose. Blood 113, 4125-4126 (2009).

28 Cifuentes RA, Murillo-Rojas J, Avella-Vargas E. Prediction of sensitivity to warfarin based on VKORC1 and CYP2C9 polymorphisms in patients from different places in Colombia. Biomedica 36(1), 91-100 (2016).

29 Duconge J, Ruaño G. Admixture and ethno-specific alleles: missing links for global pharmacogenomics. Pharmacogenomics 17(14), 1479-1482 (2016).

30 Cavallari L, Langaee TY, Momary K et al. Genetic and clinical predictors of warfarin dose requirements in African Americans. Clin. Pharmacol. Ther. 87, 459-64 (2010).

31 Perera MA, Cavallari LH, Limdi NA et al. Genetic variants associated with warfarin dose in African-American individuals: a genome-wide association study. Lancet 382(9894), 790-796 (2013).

32 Limdi NA, Brown TM, Yan Q et al. Race influences warfarin dose changes associated with genetic factors. Blood 126(4), 539-545 (2015).

33 Daneshjou R, Gamazon ER, Burkley B et al. Genetic variant in folate homeostasis is associated with lower warfarin dose in African Americans. Blood 124(14), 2298-2305 (2014). 
34 Pirmohamed M, Burnside G, Eriksson N et al. A randomized trial of genotype-guided dosing of warfarin. N. Engl. J. Med. 369, 2294-2303 (2013).

35 Zineh I, Pacanowski M, Woodcock J. Pharmacogenetics and coumarin dosing-recalibrating expectations. N. Engl. J. Med. 69(24), 2273-2275 (2013).

36 Napoli N. Genetically guided warfarin dosing lowers risk of some adverse events. Adding genotyping may be better than clinically guided dosing alone. American College of Cardiology Press Release (2017). www.acc.org/about-acc/press-releases/2017/03/18/08/47/sun-1045 am-genetically-guided-warfarin-dosing-lowers-risk-of-some-adverse-events

37 Scott SA, Lubitz SA. Warfarin pharmacogenetic trials: is there a future for pharmacogenetic-guided dosing. Pharmacogenomics 15(6), 719-722 (2014).

38 Santos PC, Marcatto LR, Duarte NE et al. Development of a pharmacogenetic-based warfarin dosing algorithm and its performance in Brazilian patients: highlighting the importance of population-specific calibration. Pharmacogenomics 16(8), 865-876 (2015).

39 Suarez-Kurtz G, Parra EJ. Population diversity in pharmacogenetics: a Latin American perspective. Adv. Pharmacol. 83(2018), 133-154 (2018).

40 Suarez-Kurtz G, Pena SDJ, Struchiner CJ, Hutz MH. Pharmacogenomic diversity among Brazilians: influence of ancestry, self-reported color, and geographical origin. Front. Pharmacol. 3, 191 (2012).

41 Bonifaz-Peña V, Contreras AV, Struchiner CJ et al. Exploring the distribution of genetic markers of pharmacogenomics relevance in Brazilian and Mexican populations. PLoS ONE 9, e112640 (2014).

42 Owusu-Obeng A, Kaszemacher T, Abul-Husn NS et al. Implementing algorithm-guided warfarin dosing in an ethnically diverse patient population using electronic health records and preemptive CYP2 C9 and VKORC1 genetic testing. Clin. Pharmacol. Ther. 100(5), 427-430 (2016). 
